\title{
$\sigma$-CENTRED FORCING AND REFLECTION OF (SUB)METRIZABILITY
}

\author{
FRANKLIN D. TALL
}

(Communicated by Andreas R. Blass)

\begin{abstract}
By using supercompact reflection and preservation lemmas for random real forcing and $\sigma$-centred forcing, we obtain a model in which every normal Moore space is submetrizable, but not every normal Moore space is metrizable.
\end{abstract}

In $\left[\mathrm{DTW}_{1}, \mathrm{DTW}_{2}\right]$ we demonstrated that Cohen and random real forcing preserve various topological properties, and used these "preservation lemmas" to establish topological reflection theorems, assuming the consistency of a supercompact cardinal. Here we shall engage in a similar exploration of $\sigma$-centred forcing. The main result we prove is

Theorem 1. Suppose it is consistent that there is a supercompact cardinal. Then it is consistent that every normal Moore space is submetrizable, but that there exists both a normal nonmetrizable Moore space and a countably paracompact Moore space that is not submetrizable.

A space is submetrizable if it has a weaker metrizable topology. Adding supercompact many Cohen or random reals gives a model in which every normal Moore space-in fact every countably paracompact Moore space $\left[\mathrm{T}_{2}, \mathrm{Bu}\right]$-is metrizable $\left[\mathrm{DTW}_{1}, \mathrm{~N}\right]$ and hence submetrizable.

The model for Theorem 1 is a simple one: let $\kappa$ be supercompact and force with the usual finite support iteration $\mathscr{P}$ of $\sigma$-centred partial orders to establish $\mathfrak{p}=\kappa$ (or, equivalently [B], $\mathrm{MA}\left(\sigma\right.$-centred) $\left.+2^{\aleph_{0}}=\kappa\right)$. It is well known that $\mathfrak{p}>$ $\aleph_{1}$ implies there is a $Q$-set implies there is a normal nonmetrizable Moore space (see, e.g., $\left[\mathrm{T}_{1}\right]$ ). Also from a $Q$-set, one gets a countably paracompact Moore space which is not submetrizable [WFR, $R_{1}$ ]. Reed and Zenor [RZ] proved that every normal Moore space of size $\leq 2^{\mathrm{N}_{0}}$ is submetrizable. Very recently, a consistent example of a normal Moore space which is not submetrizable has been constructed [STW]. Our plan is to build a model for $\mathfrak{p}=2^{\aleph_{0}}>\aleph_{1}$ in which enough reflection will hold so that the submetrizability of all normal Moore

Received by the editors January 2, 1992 and, in revised form, August 25, 1992.

1991 Mathematics Subject Classification. Primary 54A35, 54E30, 54E35, 03E35, 03E55; Secondary 54D15, 03E50.

Key words and phrases. Supercompact, reflection, random reals, $\sigma$-centred forcing, normal Moore space, submetrizable, nonmetrizable, countably paracompact.

The author acknowledges support from NSERC grant A-7354. 
spaces of cardinality $<2^{\aleph_{0}}$ will imply the submetrizability of all normal Moore spaces. As the reader of [DTW 1$]$ knows, the key to proving such reflection results is to prove preservation lemmas for the partial order $j(\mathscr{P}) / \mathscr{P}$ (assuming that it is defined), where $\mathscr{P}$ is the partial order forced with; and $j$ is an elementary embedding with critical point $\kappa$ obtained from a supercompact $\kappa$. It is relatively easy to prove that $\sigma$-centred forcing preserves nonsubmetrizablity of normal Moore spaces (Lemma 3), which is the preservation lemma one might expect to need in this context. The problem however is that $j(\mathscr{P}) / \mathscr{P}$ is not $\sigma$ centred, but only an iteration of $\sigma$-centred partial orders. The way I originally dealt with this problem was elegantly corrected and simplified by Alan Dow, who proved Lemma 2.

Definition. An $\alpha$-stage iteration $\mathscr{P}$ with finite support and typical stage $\mathscr{P}_{\beta+1}=$ $\mathscr{P}_{\beta} * \dot{\mathscr{Q}}_{\beta}$ is an absolute $\sigma$-centred iteration if in any forcing extension, for $\beta<\alpha$, $\Vdash_{\beta} \dot{\mathscr{Q}}_{\beta}$ is $\sigma$-centred.

Any iteration of $\sigma$-centred partial orders one is liable to come across will almost certainly be absolute, but one can cook up examples of nonabsolute ones by, for example, defining $q \leq q^{\prime}$ iff $\mathrm{CH}$, for all $q, q^{\prime} \in \mathscr{Q}_{\beta}$.

Lemma 2. Suppose $\Phi(X)$ is preserved by $\sigma$-centred forcing. Let $\kappa$ be a cardinal. Suppose there is a partial order $\mathscr{R}$ which forces $\kappa \leq 2^{\aleph_{0}}$ and which preserves both $\Phi(X)$ and $\sim \Phi(X)$. Then $\Phi(X)$ is preserved by finite support $\kappa$-stage absolute $\sigma$-centred iterations.

Proof. Let $\mathscr{P}$ be a finite support $\kappa$-stage absolute $\sigma$-centred iteration. Suppose $\mathscr{P}$ does not preserve $\Phi(X)$. Then $\mathscr{P} \times \mathscr{R}$ does not preserve $\Phi(X)$. Then neither does $\mathscr{R} \times \mathscr{P}$. But $\mathscr{R}$ forces that $\kappa \leq 2^{\aleph_{0}}$, while $\mathscr{P}$ is still a finite support $\kappa$-stage iteration of $\sigma$-centred partial orders, so $\mathscr{R}$ forces $\mathscr{P}$ is $\sigma$-centred, since it is well known (proved by the same method that proves the product of $\leq 2^{\aleph_{0}}$ separable spaces is separable) that the finite support iteration of $\leq 2^{\aleph_{0}} \sigma$-centred partial orders is $\sigma$-centred. But then we have a contradiction.

Remark. Note that the requirements on preservation of $\Phi$ and $\sim \Phi$ are not actually symmetric; we only need $\mathscr{R}$ preserves $\Phi(X)$ over the ground model, but we need $\mathscr{R}$ preserves $\sim \Phi$ over $V^{\mathscr{P}}$.

Thus a property $\Phi(X)$ preserved by $\sigma$-centred forcing will be preserved by $\kappa$-stage absolute $\sigma$-centred iteration if we can find some way of adding $\kappa$ reals (or, for that matter, collapsing $\kappa$ to $\omega_{1}$ ) that preserves both $\Phi(X)$ and $\sim \Phi(X)$. Submetrizability is preserved by any extension; we shall show that nonsubmetrizability of first countable spaces is preserved by adding random reals (Lemma 4, below). I do not have a direct proof that Cohen reals preserve, but it follows from Lemma 2 that they do, since Cohen real forcing is obviously an absolute $\sigma$-centred iteration. Let us also observe that the usual $\kappa$-stage iteration $\mathscr{P}$ forcing $\mathfrak{p}=\kappa$ is also an absolute $\sigma$-centred iteration. A typical stage $\mathscr{P}_{\alpha+1}=\mathscr{P}_{\alpha} * \dot{\mathscr{Q}}_{\alpha}$, where, in $V^{\mathscr{P}_{\alpha}}, \mathscr{C}$ is a collection of subsets of $\omega$ with every finite intersection infinite, $\mathscr{Q}_{\alpha}$ consists of $\left\{\langle H, \mathscr{H}\rangle: H \in[\omega]^{<\omega}\right.$, $\left.\mathscr{H} \in[\mathscr{C}]^{<\omega}\right\}$, where $\left\langle H^{\prime}, \mathscr{H}^{\prime}\right\rangle \leq\langle H, \mathscr{H}\rangle$ if $H^{\prime} \supseteq H, \mathscr{H}^{\prime} \supseteq \mathscr{H}$, and for each $C \in \mathscr{H}, H^{\prime}-C=H-C$. Note that without loss of generality, we may take the first components to actually be in $V$, rather than just names for elements 
of $V$. Note that a nice $\mathscr{P}_{\alpha}$-name $\dot{\mathscr{C}}$ for a collection of subsets of $\omega$ will still be a $\mathscr{P}_{\alpha}$-name for a collection of subsets of $\omega$ after we do some forcing over $V$, and if $\mathscr{P}_{\alpha}$ forced it to have every finite intersection infinite, it still will do so. Any finite number of conditions with the same first element have a lower bound, regardless of what model we are in, so we get the required absoluteness. It is also easy to see that if $\kappa$ is the critical point of $j$, then $\mathscr{P}$ is indeed an initial segment of $j(\mathscr{P})$. It follows that $j(\mathscr{P}) / \mathscr{P}$ is also an absolute $\sigma$-centred iteration. Of course we are being somewhat sloppy-what we really mean is that if we force with $\mathscr{P}$ over any model of ZFC, then in the resulting extension, the interpretation of $j(\mathscr{P}) / \mathscr{P}$ will be an absolute $\sigma$-centred iteration.

Let us briefly review the scheme for applying supercompact reflection in the case at hand. For details, see $\left[\mathrm{DTW}_{1}\right]$. We claim that in $V^{\mathscr{P}}$ every normal Moore space is submetrizable. Let $X$ be a normal Moore space of cardinality $\lambda$. We take $j(\kappa)>\lambda$ sufficiently large and consider the copy $j^{\prime \prime} X$, which is a subspace of $j(X)$ in $M^{j(\mathscr{P})}$. It is a normal Moore space since $j(X)$ is and that property is hereditary. Its cardinality is $\left\langle 2^{\aleph_{0}}\right.$ so it is submetrizable. Therefore it and $X$ are submetrizable in $V^{j(\mathscr{P})}$. It suffices to show that $j(\mathscr{P}) / \mathscr{P}$ preserves nonsubmetrizability; by Lemma 2 it therefore suffices to show that $\sigma$-centred forcing and random real forcing both preserve nonsubmetrizability. The $\sigma$-centred case is straightforward, so we do it first. For the random real one, we shall assume some familiarity with the Boolean approach to forcing, as in, e.g., [J].

For normal Moore spaces there is a characterization $\left[\mathbf{R}_{2}\right]$ of submetrizability which is easy to work with: there is a $\sigma$-disjoint separating open cover. An open cover $\mathscr{U}$ is said to be separating if for each $x,\{x\}=\bigcap\{U \in \mathscr{U}: x \in U\}$.

Lemma 3. $\sigma$-centred forcing preserves nonsubmetrizability of normal Moore spaces.

Proof. Let $\mathscr{P}=\langle P, \leq\rangle$ be $\sigma$-centred, i.e., $P=\bigcup_{n<\omega} P_{n}$, each $F \in\left[P_{n}\right]^{<\omega}$ has a lower bound in $P$. Let $\{\mathscr{U}(k, \alpha): k<\omega, \alpha<|X|\}, \mathscr{U}(k, \alpha) \cap \mathscr{U}(k, \beta)=0$ for $\alpha \neq \beta$, be a $\sigma$-disjoint separating open cover in the extension; we shall produce such a cover in the ground model. We shall let elements of the ground model be names for themselves in the extension. Let

$$
W_{n k \alpha}=\bigcup\left\{V: V \text { is open and }\left(\exists p \in P_{n}\right)(p \Vdash V \subseteq \dot{\mathscr{U}}(k, \alpha))\right\} .
$$

Claim $\mathscr{W}=\left\{W_{n k \alpha}: n, k<\omega, \alpha<|X|\right\}$ is the desired cover. It is certainly an open cover since $\mathscr{U}$ is. Claim $W_{n k \alpha} \cap W_{n k \alpha^{\prime}}=0$ for $\alpha \neq \alpha^{\prime}$. For if $x \in W_{n k \alpha} \cap W_{n k \alpha^{\prime}}$, there are $p, p^{\prime} \in P_{n}$ and open $V \subseteq W_{n k \alpha}$ and $V^{\prime} \subseteq W_{n k \alpha^{\prime}}$, both containing $x$, such that $p \Vdash V \subseteq \dot{\mathscr{U}}(k, \alpha)$ and $p^{\prime} \Vdash V^{\prime} \subseteq \dot{\mathscr{U}}\left(k, \alpha^{\prime}\right)$. Take $p^{\prime \prime}$ below $p$ and $p^{\prime}$. Then $p^{\prime \prime} \Vdash x \in \dot{\mathscr{U}}(k, \alpha) \cap \dot{\mathscr{U}}\left(k, \alpha^{\prime}\right)$, a contradiction. Next, to show $\mathscr{W}$ is separating, given $x \neq y$, there are $n, k, \alpha, p, V$ such that $p \in P_{n}$ and $p \vDash x \in V \subseteq \dot{\mathscr{U}}(k, \alpha) \& y \notin \dot{\mathscr{U}}(k, \alpha)$. Then $y \notin W_{n k \alpha}$, else there would be a $p^{\prime} \in P_{n}$ forcing $y \in \mathscr{U}(k, \alpha)$. But $p^{\prime}$ is compactible with $p$, a contradiction.

Lemma 4. Suppose $X$ is a first countable space in $V$. If $X$ is submetrizable in some random real extension, it is submetrizable in $V$.

Proof. As noted by Alan Dow, it suffices to prove this for adjoining more than $|X|$ random reals, since, as observed earlier, submetrizability is preserved by 
forcing. Denote by $\mathscr{T}(G)$ the topology $\mathscr{T}$ generates in the extension. Suppose $\mathscr{S} \subseteq \mathscr{T}(G)$ such that $\langle X, \mathscr{S}\rangle$ is metrizable. As will be seen shortly, we want $\mathscr{S}$ to have a non-Archimedean metric, i.e., $d(x, z) \leq \max \{d(x, y), d(y, z)\}$ for all $x, y, z \in X$. Since $X$ is forced to have cardinality $<2^{\aleph_{0}}$, it suffices to establish that all metrizable spaces of cardinality $<2^{\aleph_{0}}$ are strongly zerodimensional, for then their large inductive dimension $=0[\mathrm{E}, 7.3 .1]$ and hence they have non-Archimedean metrics $[\mathrm{E}$, 7.3.F]. By [E, 6.2.4] it suffices to show that if $A, B \subseteq X$ are such that there is an $f: X \rightarrow[0,1]$ such that $f(A) \subseteq$ $\{0\}$ and $f(B) \subseteq\{1\}$, then there is a clopen $U \supseteq A, U \cap B=0$. But take $r \in[0,1]-f^{\prime \prime} X$ and let $U=f^{-1}([0, r))=f^{-1}([0, r])$.

Without loss of generality we will take a non-Archimedean metric $d$ for $\langle X, \mathscr{S}\rangle$ such that $d(x, y) \leq 1$ for all $x, y \in X$. Now define in $V$,

$$
\rho(x, y)=\sum_{n \in N} \frac{\mu(\|\dot{d}(x, y)>1 / n\|)}{2^{n}},
$$

where $N=\omega-\{0\}, \mu$ is the measure on the measure algebra, say $\mathscr{B}_{\kappa}$, that we are forcing with, and $\|\Phi\|$ denotes the Boolean value of the statement $\Phi$. We shall use $+, \cdot, \sum, \prod$ to denote the Boolean operations. Claim $\rho$ is a metric on $X$ such that the topology it induces is weaker than $\mathscr{T}$, i.e., $\langle X, \mathscr{T}\rangle$ is submetrizable. Certainly $\rho$ is symmetric and $\rho(x, x)=0 . \quad \rho(x, y)=0$ implies $x=y$ since $x \neq y$ implies $\|\dot{d}(x, y)>0\|=1$. To prove the triangle inequality, note that since $d$ is non-Archimedean, $d(x, z)>1 / n$ implies $d(x, y)>1 / n$ or $d(y, z)>1 / n$, so

$$
\|\dot{d}(x, z)>1 / n\| \leq\|\dot{d}(x, y)>1 / n\|+\|\dot{d}(x, z)>1 / n\| .
$$

Then

$$
\begin{aligned}
\mu(\|\dot{d}(x, z)>1 / n\|) & \leq \mu(\|\dot{d}(x, y)>1 / n\|+\|\dot{d}(y, z)>1 / n\|) \\
& \leq \mu(\|\dot{d}(x, y)>1 / n\|)+\mu(\|\dot{d}(y, z)>1 / n\|) .
\end{aligned}
$$

Thus,

$$
\sum_{n \in N} \frac{\mu(\|\dot{d}(x, z)>1 / n\|)}{2^{n}} \leq \sum_{n \in N} \frac{\mu(\|\dot{d}(x, y)>1 / n\|)}{2^{n}}+\sum_{n \in N} \frac{\mu(\|\dot{d}(y, z)>1 / n\|)}{2^{n}},
$$

i.e., $\rho(x, z) \leq \rho(x, y)+\rho(y, z)$, which was to be proved.

Finally, suppose $F$ is closed in the topology induced by $\rho$. Claim $F$ is closed in $\mathscr{T}$. It suffices to show that if $x \in \bar{F}$ in $\mathscr{T}$, then $\rho(x, F)=0$. $x \in \bar{F}$ in $\mathscr{T}$ implies there is a sequence $\left\{x_{n}\right\}_{n<\omega}$ from $F$ converging to $x$ in $\mathscr{I}$. Then $\left\{x_{n}\right\}_{n<\omega} \rightarrow x$ in $\mathscr{T}(G)$. Then $\left\{x_{n}\right\}_{n<\omega} \rightarrow x$ in $\mathscr{S}$ so without loss of generality $1 \Vdash \dot{d}\left(x, x_{n}\right) \rightarrow 0$, so for every $k \in \mathrm{N}$,

$$
\left\|(\exists n)(\forall m \geq n)\left(\dot{d}\left(x_{m}, x\right)<1 / k\right)\right\|=\mathbf{1},
$$

so

$$
\sum_{n \in N} \prod_{m \geq n}\left\|\dot{d}\left(x_{m}, x\right)<1 / k\right\|=\mathbf{1} .
$$

Fix $\varepsilon>0$ and pick $i$ such that $\sum_{n \geq i} 1 / 2^{n}<\varepsilon / 2$. Pick $j$ such that

$$
\mu\left(\sum_{n \leq j} \prod_{m \geq n}\left\|\dot{d}\left(x_{m}, x\right)<1 / i\right\|\right)>1-\varepsilon / 2 .
$$


Note that for $p \leq j$,

$$
\prod_{m \geq p}\left\|\dot{d}\left(x_{m}, x\right)<\frac{1}{i}\right\| \leq \prod_{m \geq j}\left\|\dot{d}\left(x_{m}, x\right)<\frac{1}{i}\right\|,
$$

so

$$
\sum_{n \leq j} \prod_{m \geq n}\left\|\dot{d}\left(x_{m}, x\right)<\frac{1}{i}\right\|=\prod_{m \geq j}\left\|\dot{d}\left(x_{m}, x\right)<\frac{1}{i}\right\| .
$$

Thus,

$$
\mu\left(\prod_{m \geq j}\left\|\dot{d}\left(x_{m}, x\right)<\frac{1}{i}\right\|\right)>1-\frac{\varepsilon}{2} .
$$

So, for each $m \geq j, \mu\left(\left\|\dot{d}\left(x_{m}, x\right)<1 / i\right\|\right)>1-\varepsilon / 2$, and hence

$$
\mu\left(\left\|\dot{d}\left(x_{m}, x\right)>1 / i\right\|\right)<\varepsilon / 2 .
$$

But for any $m$, in particular for $m \geq j$ and $i$,

$$
\begin{aligned}
\sum_{n \in N} \frac{\mu\left(\left\|\dot{d}\left(x_{m}, x\right)>1 / n\right\|\right)}{2^{n}}= & \sum_{n<m} \frac{\mu\left(\left\|\dot{d}\left(x_{m}, x\right)>1 / n\right\|\right)}{2^{n}} \\
& +\sum_{n \geq m} \frac{\mu\left(\left\|\dot{d}\left(x_{m}, x\right)>1 / n\right\|\right)}{2^{n}}
\end{aligned}
$$

Now

$$
\begin{aligned}
\sum_{n<i} \frac{\mu\left(\left\|\dot{d}\left(x_{m}, x\right)>1 / n\right\|\right)}{2^{n}} & \leq \sum_{n<i} \frac{\mu\left(\left\|\dot{d}\left(x_{m}, x\right)>1 / i\right\|\right)}{2^{n}} \\
& \leq \mu\left(\left\|\dot{d}\left(x_{m}, x\right)>\frac{1}{i}\right\|\right)<\frac{\varepsilon}{2}
\end{aligned}
$$

so

$$
\sum_{n \in N} \frac{\mu\left(\left\|\dot{d}\left(x_{m}, x\right)>1 / n\right\|\right)}{2^{n}}<\frac{\varepsilon}{2}+\frac{\varepsilon}{2}=\varepsilon,
$$

thus indeed $\left\{x_{n}\right\}_{n<\omega} \rightarrow \dot{x}$ in the $\rho$-topology, so $\rho(x, F)=0$.

Remark. In [DTW 2 ] we showed that if supercompact many Cohen or random reals are adjoined to a model of set theory, then first countable spaces are metrizable if all their subspaces of size less than $2^{\aleph_{0}}$ are. That proof used the characterization of metrizable spaces as collectionwise normal Moore spaces. Our methods here provide a different proof for the random real case. Follow the scheme of the proof of Lemma 4 but assume $\mathscr{S}=\mathscr{T}(G)$. It remains to show that a set $F$ closed in $\mathscr{T}$ is $\rho$-closed. Suppose $\rho(x, F)=0$, claim $x \in F$. It suffices to show some $p \Vdash \dot{d}(x, F)=0$, for then $p \Vdash x \in F$, since $F$ is closed in $\mathscr{T}(G)=\mathscr{S}$, and so by absoluteness, $x$ really is in $F$. If there is no such $p,\|\dot{d}(x, F)>0\|=1$ so

$$
\sum_{n \in N}\left\|\dot{d}(x, F)>\frac{1}{n}\right\|=1 .
$$


Therefore

$$
\sum_{n \in N} \prod_{y \in F}\left\|\dot{d}(x, y)>\frac{1}{n}\right\|=1 .
$$

Fix $\varepsilon, 0<\varepsilon<1$. There is a $k \in N$ such that

$$
\mu\left(\sum_{1 \leq n \leq k} \prod_{y \in F}\left\|\dot{d}(x, y)>\frac{1}{n}\right\|\right)>1-\varepsilon .
$$

Therefore

$$
\sum_{1 \leq n \leq k} \mu\left(\prod_{y \in F}\left\|\dot{d}(x, y)>\frac{1}{n}\right\|\right)>1-\varepsilon .
$$

Then for each $y \in F$,

$$
\sum_{1 \leq n \leq k} \mu\left(\left\|\dot{d}(x, y)>\frac{1}{n}\right\|\right)>1-\varepsilon .
$$

Now for any $y$,

$$
\begin{aligned}
\sum_{1 \leq n \leq k} \frac{\mu(\|\dot{d}(x, y)>1 / n\|)}{2^{n}} & \geq \sum_{1 \leq n \leq k} \frac{\mu(\|\dot{d}(x, y)>1 / n\|)}{2^{k}} \\
& =\frac{1}{2^{k}} \sum_{1 \leq n \leq k} \mu\left(\left\|\dot{d}(x, y)>\frac{1}{n}\right\|\right)>\frac{1-\varepsilon}{2^{k}} .
\end{aligned}
$$

But since $\rho(x, F)=0$, there is a $y \in F$ such that

$$
\sum_{n \in N} \frac{\mu(\|\dot{d}(x, y)>1 / n\|)}{2^{n}}<\frac{1-\varepsilon}{2^{k}},
$$

so

a contradiction.

$$
\sum_{1 \leq n \leq k} \frac{\mu(\|\dot{d}(x, y)>1 / n\|)}{2^{n}}<\frac{1-\varepsilon}{2^{k}},
$$

An advantage of this proof over the one in $\left[\mathrm{DTW}_{2}\right]$ is that it enables us to prove reflection theorems for different classes of "nearly metrizable" spaces, e.g.,

Theorem 5. If it is consistent that there is a supercompact cardinal, it is consistent that if a first countable space has all subspaces of size $<2^{\aleph_{0}}$ semimetrizable, then it is semimetrizable.

We leave the proof to the reader, as well as the variations using other properties near metrizability which can be found in $\$ \S 9$ and 10 of [G]. Of course we have implicitly proved the submetrizable version. I do not see how to prove that nonsubmetrizability is preserved by random or Cohen extensions, using the methods of $\left[\mathrm{DTW}_{2}\right]$. The problem is that the metrizable topology $\mathscr{S} \subseteq \mathscr{T}(G)$ cannot be assumed to have a basis in the ground model. I suspect the method of defining a function in $V$ from a function in a random real extension may have other applications; one could also try using integrals instead of sums, e.g.,

$$
\phi(\overrightarrow{\mathbf{x}})=\int_{0}^{\infty} \frac{\mu(\|\dot{f}(\overrightarrow{\mathbf{x}})>1 / r\|)}{r^{2}} d r .
$$


Note that then

$$
g(\overrightarrow{\mathbf{x}})=\int_{0}^{\infty} \frac{\mu(\|g(\overrightarrow{\mathbf{x}})>1 / r\|)}{r^{2}} d r,
$$

for $g$ a nonnegative real-valued function in $V$. (D. Gautreau observed that $\phi$ may not be defined unless $f$ is forced to be bounded.)

Having established Theorem 1, we give another application of our methods. The following result originally appeared in an early draft of [ $\left.\mathrm{T}_{4}\right]$ but is better placed here.

Theorem 6. If it is consistent that there is a supercompact cardinal, it is consistent that

(1) there is a first countable space which is

(a) $\lambda$ - $\lambda$-collectionwise normal (i.e., discrete collections of $\leq \lambda$ sets, each of size $\leq \lambda$ can be separated) for all $\lambda<2^{\aleph_{0}}$,

(b) such that all subspaces of size $<2^{\aleph_{0}}$ are metrizable,

(c) not collectionwise normal, (and hence)

(d) not metrizable.

But

(2) for any normal space $X$ in which each point has a neighbourhood base of cardinality $<2^{\aleph_{0}}$, if $X$ is countably metacompact, it cannot satisfy both (a) and $(\mathrm{c})$, while if closed sets are $G_{\delta}$ 's, it cannot satisfy both (b) and (d).

Proof. We use the same model as before. The space in question is constructed in [vD] from $\mathfrak{p}=2^{\aleph_{0}}$. By the same method as before, it suffices to prove:

Lemma 7. (i) Random (or Cohen) forcing preserves noncollectionwise normality.

(ii) Random (or Cohen) forcing preserves nonmetrizability.

(iii) $\sigma$-centred forcing preserves noncollectionwise normality in normal countably metacompact spaces.

(iv) $\sigma$-centred forcing preserves nonmetrizability in perfectly normal spaces.

(i) is proved in $\left[D T W_{1}\right]$ and (ii) in $\left[D^{2} W_{2}\right]$. We omit (iii), since it is really just the proof that screenable normal spaces are collectionwise normal with respect to countably metacompact closed sets (see, e.g., $\left[\mathrm{T}_{3}\right]$ ). The point is that the usual argument converts a separation in the extension into a ground model collection of countably many partial separations with union covering the whole discrete collection (which may be assumed to consist of closed sets). For (iv), we proceed exactly as in Lemma 3 to bring down a $\sigma$-disjoint collection which is a base-rather than a separating open cover-in the extension. Claim the resulting $\bigcup_{n<\omega} \mathscr{W}_{n}$ obtained in the ground model will also be a base. Once we prove that, we will be done, since perfectly normal spaces with $\sigma$-disjoint bases are metrizable [A]. Given then an open $S$ in the ground model, and $x \in S$, there are $p, k, \alpha$ such that $p \Vdash x \in \dot{\mathscr{U}}(k, \alpha) \subseteq S$. There is an $n$, a $p^{\prime} \leq p$, $p^{\prime} \in P_{n}$, and an open $V$ such that $p^{\prime} \Vdash x \in V \subseteq \dot{\mathscr{U}}(k, \alpha) \subseteq S$. But then $x \in V \subseteq S$; in fact $V \subseteq W_{n k \alpha} \subseteq S$. The former inclusion is by definition of $W_{n k \alpha}$; to see the latter, if $y \in W_{n k \alpha}$, there is a $p^{\prime \prime} \in P_{n}$ and a $V^{\prime}$ such that $p^{\prime \prime} \Vdash y \in V^{\prime} \subseteq \dot{\mathscr{U}}(k, \alpha)$. Take $p^{\prime \prime \prime}$ below $p^{\prime}$ and $p^{\prime \prime}, p^{\prime \prime \prime} \Vdash y \in V^{\prime} \subseteq$ $\dot{\mathscr{U}}(k, \alpha) \subseteq S$, so $y \in S$.

"Perfectly normal" cannot be weakened to "normal" in (iv): Dow [D] has shown that $\mathfrak{p}=2^{\aleph_{0}}>\aleph_{1}$ implies there is a Lindelöf normal first countable 
nonmetrizable space of cardinality $2^{\aleph_{0}}$, in which subspaces of size $<2^{\aleph_{0}}$ are metrizable. It cannot be weakened to "perfect" either: van Douwen's space is a Moore space but Fleissner [F] has observed that the adjunction of one dominating real makes it metrizable. Such adjunction is $\sigma$-centred. For the same reason, "normal" cannot be dropped in (iii).

\section{REFERENCES}

[A] C. E. Aull, Some base axioms for topology involving enumerability, General Topology and Its Relations to Modern Analysis and Algebra (Proc. Kanpur Topology Conf., 1968), Academia, Prague, 1971, pp. 54-61.

[B] M. Bell, On the combinatorial principle P(c), Fund. Math. 114 (1981), 149-157.

[Bu] D. K. Burke, PMEA and first countable, countably paracompact spaces, Proc. Amer. Math. Soc. 92 (1984), 455-460.

[vD] E. K. van Douwen, The integers and topology, Handbook of Set-Theoretic Topology (K. Kunen and J. E. Vaughan, eds.), North-Holland, Amsterdam, 1984, pp. 111-167.

[D] A. Dow, An empty class of nonmetric spaces, Proc. Amer. Math. Soc. 104 (1988), 999-1001.

[DTW1] A. Dow, F. D. Tall, and W. Weiss, New proofs of the consistency of the normal Moore space conjecture. I, Topology Appl. 37 (1990), 33-51.

[DTW2] _ New proofs of the consistency of the normal Moore space conjecture. II, Topology Appl. 37 (1990), 115-130.

[E] R. Engelking, General topology, Heldermann-Verlag, Berlin, 1989.

[F] W. Fleissner, Forcing and topological properties, preprint.

[G] G. Gruenhage, Generalized metric spaces, Handbook of Set-Theoretic Topology (K. Kunen and J. E. Vaughan, eds.), North-Holland, Amsterdam, 1984, pp. 423-501.

[J] T. Jech, Set theory, Academic Press, New York, 1978.

[N] P. J. Nyikos, A provisional solution to the normal Moore space problem, Proc. Amer. Math. Soc. 78 (1980), 429-435.

[R1] G. M. Reed, On normality and countable paracompactness, Fund. Math. 110 (1980), 145-152.

[R2] _ Set-theoretic problems in Moore spaces, Open Problems in Topology (J. van Mill and G. M. Reed, eds.), North-Holland, Amsterdam, 1990, pp. 163-181.

[RZ] G. M. Reed and P. L. Zenor, Metrization of Moore spaces and generalized manifolds, Fund. Math. 91 (1976), 213-220.

[STW] D. Shakhmatov, F. D. Tall, and S. Watson, A normal Moore space which is not submetrizable (in preparation).

[T1] F. D. Tall, Set-theoretic consistency results and topological theorems concerning the normal Moore space conjecture and related problems, Thesis, Univ. of Wisconsin, Madison, 1969, Dissertationes Math. (Rozprawy Mat.) 148 (1977), 1-53.

[T2] Countably paracompact Moore spaces are metrizable in the Cohen model, Topology Proc. 9 (1984), 145-148.

[T3] _ Normality versus collectionwise normality, Handbook of Set-Theoretic Topology (K. Kunen and J. E. Vaughan, eds.), North-Holland, Amsterdam, 1984, pp. 685-732.

[T4] _ Topological applications of generic huge embeddings, Trans. Amer. Math. Soc. 341 (1994), 45-68.

[WFR] M. L. Wage, W. G. Fleissner, and G. M. Reed, Countable paracompactness in perfect spaces, Bull. Amer. Math. Soc. 82 (1976), 635-639.

Department of Mathematics, University of Toronto, Toronto, Canada M5S 1A1

E-mail address: tall@math.toronto.edu 